\title{
461 UPDATED PFS ANALYSIS OF TORIPALIMAB WITH ANLOTINIB AND CHEMOTHERAPY AS FIRST-LINE THERAPY IN PATIENTS WITH EXTENSIVE-STAGE SMALL- CELL LUNG CANCER (ES-SCLC)
}

Hao Luo*, Dan Jian, Yan Feng, Li Zhong, Qian Chen, Wei Guan, Shiheng Zhang, Jiamin Luo, Xueqin Yang, Kan Gong, Yanli Xiong, Mengxia Li, Mingfang Xu, Yu Pu, Liang Zhao, Chengyuan Qian, Nan Dai, Dong Wang. Cancer Center of Daping Hospital, Army Medical Center, Chongqing, China

Background This trail is an open-label, single-arm, phase II study that aims to observe the efficacy and safety of toripalimab combined with anlotinib and platinum-etoposide (EP) chemotherapy as first-line treatment in ES-SCLC (Clinical trial information: NCT04731909). The preliminary results of the study have been presented in 2020 ASCO abstract e20570, which demonstrated $100 \%$ objective response rate (ORR) and tolerable safety. Here we report PFS analysis results of the study.

Methods The study enrolled treatment-naïve ES-SCLC patients (18-75 years, ECOG PS $\leq 2)$ who have measurable target lesion evaluated by RECIST v1.1. All enrolled patients received toripalimab (240 $\mathrm{mg}$, d1) combined with etoposide $(100 \mathrm{mg} / \mathrm{m} 2, \mathrm{~d} 1-3)$ plus carboplatin $(\mathrm{AUC}=5, \mathrm{~d} 1) /$ cisplatin $(75$ $\mathrm{mg} / \mathrm{m} 2, \mathrm{~d} 1)$ and anlotinib (12 $\mathrm{mg} \mathrm{QD}, \mathrm{d} 1-14)$ of a 21-day cycle. After 4-6 cycles of the treatment, patients who achieved complete response (CR), partial response (PR) or stable disease (SD) could continue to receive maintenance therapy with toripalimab and anlotinib until disease progression. The primary endpoint was overall survival (OS). ORR, disease control rate (DCR), progression-free survival (PFS) and safety were set as secondary endpoints.

Results As of July 16, 2021, the median follow-up was 13.7 months. 9 disease progression events occurred of the enrolled 16 treatment-naïve ES-SCLC patients (14 males, 2 females, median age 63). The investigator-assessed median PFS was 13.3 months (95\%CI: 5.0-21.6). The PFS rate at 6 months was $81.3 \%$ and the PFS rate at 12 months was $31.3 \% .15$ patients were still alive and the study treatments for 7 patients were still ongoing. At the data cutoff, there was only 1 patient dead with 37.6 months OS and the median OS was not reached. No new unexpected adverse events were observed.

Conclusions Combined with preliminary data at 2020 ASCO, toripalimab combined with anlotinib and EP chemotherapy showed excellent ORR and PFS as well as tolerable safety in treatment-naive ES-SCLC. The combination therapy is expected to provide clinically meaningful OS benefit and become a promising treatment option.

Trial Registration This study is registered with ClinicalTrials. gov (National Institutes of Health), number NCT04731909.

Ethics Approval The program was approved by the ethics committee of Army Medical Center (Daping Hospital ).

http://dx.doi.org/10.1136/iitc-2021-SITC2021.461 